\title{
Regards sur la situation de la morphologie en France
}

\author{
Fradin Bernard \\ LLF, CNRS \& Université Paris 7-Denis Diderot \\ bernard.fradin@linguist.jussieu.fr \\ Dal Georgette \\ Université de Lille 3 \& STL, CNRS \& U Lille 1 \& Lille 3 \\ georgette.dal@univ-lille3.fr
}

La présentation qui suit énumère les principaux thèmes qui devraient être abordés à la présentation générale de la table ronde consacrée à la morphologie lors du Congrès mondial de linguistique française. Une bibliographie sommaire est fournie pour chaque point, avec un tri entre les références qui concernent plus spécifiquement le domaine du français et les autres.

\subsubsection{Les données}

L'accès massif à des données provenant de corpus ou de ressources numériques a changé radicalement la donne en morphologie, tant au plan des hypothèses qu'on peut faire que de leur falsification. Il s'agit d'un cas clair où le quantitatif induit un saut qualitatif. On se penchera sur les nouvelles problématiques induites par l'accès massif à des données attestées, sur les problèmes que celles-ci soulèvent, sur le crédit à accorder aux validations qu'elles permettent. L'équilibre à trouver entre les données issues de ressources numériques et les données provenant de l'intuition sera aussi discuté. On évoquera enfin la place et poids des données externes (psycholinguistiques essentiellement).

\section{Général}

Dressler Wolfgang U. 1994. "Evidence from the first stages of morphology acquisition for linguistic theory: extragrammatic morphology and diminutives". Acta Linguistica Hafniensia 27 1:91-108.

Plag Ingo, Christiane Dalton-Puffer \& Harald R. Baayen. 1999. "Morphological Productivity across speech and writing". English Language and Linguistics 3:209-228.

Thornton Anna. 1999. "On Italian derivatives with antesuffixal glides". Yearbook of Morphology 1998:103-126.

\section{Domaine français}

Dal Georgette. 2003. "Productivité morphologique: définitions et notions connexes". Langue française 140:3-37.

Fradin Bernard, Georgette Dal, Natalia Grabar, Stéphanie Lignon, Fiammetta Namer, Delphine Tribout \& Pierre Zweigenbaum. 2007. "Remarques sur l'usage des corpus en morphologie". Langages 167:00-00.

Grabar Natalia, Georgette Dal, Bernard Fradin, Nabil Hathout, Stéphanie Lignon, Fiammetta Namer, Clément Plancq, Delphine Tribout, François Yvon \& Pierre Zweigenbaum. 2006. "Productivité quantitative de la suffixation par -Able dans un corpus journalistique du français". In 
Lexicometra. $8^{e} J A D T$, Viprey Jean-Marie (ed). 473-485. Besançon: Presses Universitaires de Franche-Comté.

Grabar Natalia, Georgette Dal, Bernard Fradin, Nabil Hathout, Stéphanie Lignon, Fiammetta Namer, Clément Plancq, Delphine Tribout, François Yvon \& Pierre Zweigenbaum. 2006. "Productivité quantitative des suffixation par -ité et -Able dans un corpus journalistique du français". In Verbum ex machina. Actes de la 13e conférence sur le TALN, Mertens Piet, Cédric Fairon, Anne Dister \& Patrick Watrin (eds). 167-175. Louvain: Presses Universitaires de Louvain.

Hathout Nabil, Marc Plénat \& Ludovic Tanguy. 2003. "Enquête sur les dérivés en -able". Cahiers de grammaire 28:49-90.

Plénat Marc \& Stéphanie Lignon. 2007 (à par.). "Echangisme suffixal et contraintes phonologiques". In Aperçus de morphologie du français, Fradin Bernard, Françoise Kerleroux \& Marc Plénat (eds). 00-00. Saint-Denis: Presses Universitaires de Vincennes.

Plénat Marc, Stéphanie Lignon, Nicole Serna \& Ludovic Tanguy. 2002. "La conjecture de Pichon". Corpus et recherches linguistiques 1:105-150.

\subsubsection{Phénomènes dont l'étude récente a fait bouger les choses}

\section{2.1. Etude de phénomènes flexionnels}

Depuis une quinzaine d'années, l'étude de phénomènes de flexion a conduit à remettre au premier plan la notion de paradigme et à mieux appréhender les propriétés de ces derniers. Des descriptions minutieuses et opératoires de paradigmes flexionnels complexes ont ainsi vu le jour (Anderson, Stump, Dressler \& Kilani-Schoch, etc.). Parallèlement, des principes structurants ont été proposés (Carstairs-McCarthy) qui visent à caractériser ce qu'est un paradigme normal et à permettre d'établir, du même coup, un inventaire raisonné des écarts que manifestent les paradigmes. Parmi ces écarts, figurent notamment le syncrétisme, la déponence, la supplétion, la défection. La vision plus claire que par le passé qu'on peut avoir de ces phénomènes résulte en partie de l'effort systématique qui a été fait pour exprimer ce qui est général dans les phénomènes de flexion et pour se donner des outils permettant de capter ces généralisations. L'importance accordée aux formes de surface, plutôt qu'à des représentations abstraites, est une autre tendance lourde qui influe sur les modèles proposés pour décrire les phénomènes flexionnels.

L'étude des phénomènes de flexion a fourni la majorité des faits empiriques et/ou méthodologiques conduisant à la remise en cause de la notion de morphème telle qu'elle avait été élaborée par les linguistes structuralistes.

\section{Général}

Baerman Matthew, Dunstan Brown \& Greville Corbett. 2005. The Syntax-Morphology Interface. A study of syncretism. Vol. 109, Cambridge Studies in Linguistics. Cambridge: Cambridge University Press.

Blevins James. 2003. "Stems and paradigms". Language 79 4:737-767.

Booij Geert E. 1997. "Autonomous morphology and paradigmatic relations". Yearbook of Morphology 1996:35-53.

Carstairs Andrew. 1987. Allomorphy in Inflexion. London: Croom Helm. 
Carstairs-McCarthy Andrew. 1998. "How lexical semantics constrains inflectional allomorphy". Yearbook of Morphology 1997:1-24.

Carstairs-McCarthy Andrew. 1998. "Paradigmatic Structures: Inflectional Paradigms and Morphological Classes". In The Handbook of Morphology, Spencer A. \& A. M. Zwicky (eds). 322-334. Oxford: Blackwell Publishers.

Corbett Greville. 2007. "Canonical typology, suppletion, and possible words". Language 83 1:8-42.

Maiden Martin. 2004. "When lexemes becomes allomorphs. On the genesis of suppletion". Folia Linguistica 38:227-256.

Stump Gregory T. 2001. Inflectional Morphology. A Theory of Paradigm Structure. Cambridge: Cambridge University Press.

Veselinova Ljuba N. 2006. Suppletion in Verb Paradigms. Amsterdam / Philadelphia: John Benjamins.

Domaine français

Bonami Olivier \& Gilles Boyé. 2002. "Suppletion and stem dependency in inflectional morphology". In Proceedings of the HPSG '01 Conference, Van Eynde Frank, Lars Hellan \& D. Beerman (eds)Stanford: CSLI Publications.

Bonami Olivier \& Gilles Boyé. 2003. "Supplétion et classes flexionnelles dans la conjugaison du français". Langages 152:102-126.

Bonami Olivier \& Gilles Boyé. 2005. "Construire le paradigme d'un adjectif". Recherches Linguistiques de Vincennes 34:77-98.

Bonami Olivier \& Gilles Boyé. 2006. "Deriving Inflectional Irregularity". In Proceedings fo the HPSG06 Conference held in Varna, Müller Stefan (ed). 0000. Stanford: CSLI Publications.

Bonami Olivier \& Gilles Boyé. 2006. "Subregular defaults in French conjugation". Paper read at 12th International Morphology Meeting, May 25-29, at Budapest.

Bonami Olivier, Gilles Boyé \& Jesse Tseng. 2005. "Sur la grammaire des consonnes latentes". Langages 158:89-100.

\section{2.2. Etude des phénomènes de composition}

La question de la composition reste l'objet de débats. La discussion porte sur le statut du phénomène (démarcation entre syntaxe et morphologie), sur la nature des constituants mis en jeu (mot, thème morphologique, élément de composition, syntagme) et sur l'inventaire des catégories qui servent à former les composés. Le nombre de langues où le phénomène de la composition fait l'objet d'une description en profondeur s'est accru. Mais l'attention portée aux faits reste variable et se trouve souvent artificiellement biaisée par les positionnements théoriques. La question de la commensurabilité des descriptions restera posée tant qu'un accord ne sera pas trouvé sur les paramètres à prendre en compte pour décrire la composition.

\section{Général}


Bauer Laurie. 2005. "The borderline between derivation and compounding". In Morphology and its Demarcations, Dressler W. U., D. Kastovsky, O. E. Pfeiffer \& F. Rainer (eds). 97-108. Amsterdam / Philadelphia: John Benjamins.

Bisetto Antonietta \& Sergio Scalise. 2005. "The classification of compounds". Lingue e linguaggio 2 2005:319-330.

Ceccagno Antonella \& Sergio Scalise. 2006. "Classification, structure and headedness of Chinese compounds". Lingue e linguaggio V 2:233-260.

Dressler Wolfgang U. 2005. "Towards a natural morphology of compounding". Linguistica XLV:29-39.

Fukushima Kazuhiko. 2005. "Lexical V-V compounds in Japanese: lexicon vs. syntax". Language 81 3:568-612.

Hacken ten Puisten. 2000. "37. Derivation and Compounding". In Morphologie Morphology. Ein internationales Handbuch zur Flexion und Wortbildung An International Handbook on Inflection and Word-Formation, Vol. 1, Booij G., C. Lehmann \& J. Mugdan (eds). 349-360. Berlin / New York: Walter de Gruyter.

Libben Gary \& Gonia Jarema (eds) 2006. The Representation and Processing of Compound Words. Oxford: Oxford University Press.

Packard Jerome L. 2000. The Morphology of Chinese. A Linguistic and Cognitive Approach. Cambridge: Cambridge University Press.

Ralli Angela. 2004. "Stem-based versus Word-based morphological configurations: the case of moderne Greek preverbs". Lingue e linguaggio 4 2:269-302.

Ralli Angela. 2007. "Compound marking in a cross-linguistic approach". In Morphologie à Toulouse. Actes du colloque international de morphologie 4ème Décembrettes, Hathout N. \& F. Montermini (eds). 207-220. München: LINCOM Europa.

Scalise Sergio \& Emiliano Guevarra. 2006. "Exocentric Compounding in a Typological Framework". Lingue e linguaggio $\mathbf{V}$ 2:185-206.

\section{Domaine français}

Corbin Danielle. 1992. "Hypothèse sur les frontières de la composition nominale". Cahiers de grammaire 17:25-55.

Dal Georgette \& Amiot Dany. 2007. "Composition néoclassique en français et ordre des constituants". In D. Amiot (éd.) La composition dans une perspective typologique, Arras, Artois Presses Université.

Fradin Bernard. (to appear). "Compounding in French". In Oxford Handbook on Compounding, Lieber Rochelle \& Pavol Štekauer (eds). 00-00. Oxford: Oxford University Press.

Lesselingue Chrystèle. 2003. "Les noms composés [NN]N 'holonymiques' : illustration de la spécificité sémantique des unités construites morphologiquement". In Les unités morphologiques / Morphological Units, Vol. 3, Silexicales, Fradin Bernard, Georgette Dal, Nabil Hathout, Françoise Kerleroux, Marc Plénat \& Michel Roché (eds). 100-107. Villeneuve d'Ascq: SILEX: CNRS \& Université Lille 3. 
Namer Fiammetta. 2007. "Composition néoclassique : est-on dans l' « hétéromorphosémie »?". In Hathout N., Montermini F. (eds), Morphologie à Toulouse. Actes du colloque international de Morphologie 4èmes Décembrettes, München, Lincom Europa (LSTL 37), pp. 185-206.

Namer Fiammetta \& Florence Villoing. 2007. "Have Cutthroat Anything to do with Trarcheotomes? Distinctive Properties of VN vs. NV Compounds in French". In On-line proceedings of the 5th Mediterranean Morphology Meeting, Booij G., B. Fradin, S. Scalise \& A. Ralli (eds). 105-124. Fréjus, 15-18 September 2005: University of Bologna. URL, http://mmm.lingue.unibo.it.

Villoing Florence. 2003. "Les mots composés VN du français: arguments en faveur d'une construction morphologique". Cahiers de grammaire 28:183-196.

Villoing Florence. 2008 (à par.). "Les mots composés VN". In Aperçus de morphologie $d u$ français, Fradin B., F. Kerleroux \& M. Plénat (eds). 00-00. Saint-Denis: Presses Universitaires de Vincennes.

\section{2.3. Etude des phénomènes de dérivation}

Les travaux détaillés sur les phénomènes dérivationnels sont considérablement moins nombreux que ceux relatifs à la flexion ou même à la composition. Si certains problèmes qu'ils posent sont en partie identifiés (compositionnalité décalée, ajustement par rapport aux exposants flexionnels, nature des bases, etc.), il reste impossible de dégager des généralisations valables pour l'ensemble des phénomènes dérivationnels.

Les phénomènes bénéficiant d'une description approfondie sont rares. Malgré cette situation, l'étude des phénomènes dérivationnels se maintient en France. Parmi ceux-ci figurent la conversion, la morphologie évaluative, certains dérivés déverbaux ou désadjectivaux.

\section{Général}

Aronoff Mark \& Sungeun Cho. 2001. "The Semantics of -ship Suffixation". Linguistic Inquiry 32 1:167-173.

Barker Chris. 1998. "Episodic -ee in English: A thematic role constraint". Language 74 4:695-727.

Kiefer Ferenc. 1992. "Hungarian Derivational Morphology, Semantic Complexity, and Semantic Markedness". In Lexical Matters, Sag I. \& A. Szabolcsi (eds). 183208. Stanford: CSLI.

Riehemann Suzanne Z. 1998. "Morphology and the Hierarchical Lexicon". Journal of Comparative Germanic Linguistics 2:49-77.

\section{Domaine français}

Amiot Dany \& Georgette Dal. 2007. "Integrating Neoclassical Combining Forms into a Lexeme-based Morphology". In On-line proceedings of the 5th Mediterranean 
Morphology Meeting, Booij G., B. Fradin, S. Scalise \& A. Ralli (eds). 323-336. Fréjus, 15-18 September 2005: University of Bologna. URL, http://mmm.lingue.unibo.it.

Amiot Dany \& Walter de Mulder. 2003. "Préposition contre préfixe". Recherches linguistiques 26:203-232.

Dal Georgette, Natalia Grabar, Stéphanie Lignon, Clément Plancq \& Delphine Tribout. 2007. "Les adjectifs en inXable du français". In La négation dans les langues romanes, Floricic F. (ed). 205-224. Amsterdam / Philadelphia: John Benjamins.

Dal Georgette. 2007. "Les adverbes de manière en -ment du français: dérivation ou flexion?" In Morphologie à Toulouse. Actes du colloque international de morphologie 4ème Décembrettes, Hathout N. \& F. Montermini (eds). 121-147. München: LINCOM Europa.

Fradin Bernard \& Françoise Kerleroux. 2008 (à par.). "L'identité lexémique". In Aperçus de morphologie du français, Fradin B., F. Kerleroux \& M. Plénat (eds). 00-00. Saint-Denis: Presses Universitaires de Vincennes.

Fradin Bernard, Françoise Kerleroux \& Marc Plénat (eds) 2008 (à par.). Aperçus de morphologie du français. Saint-Denis: Presses Universitaires de Vincennes.

Fradin Bernard. 2007. "Three puzzles about denominal adjectives in -eux". Acta Linguistica Hungarica 54 1:3-32.

Fradin Bernard. 2008. "Les adjectifs relationnels et la morphologie". In La raison morphologique. Hommage à la mémoire de Danielle Corbin, Fradin B. (ed). 69-91. Amsterdam / Philadelphia: John Benjamins.

Kerleroux Françoise. 1996. La coupure invisible. Lille: Presses Universitaires du Septentrion.

Kerleroux Françoise. 2008. "Des noms indistincts". In La raison morphologique. Hommage à la mémoire de Danielle Corbin, Fradin B. (ed). 113-132. Amsterdam / Philadelphia: John Benjamins.

\subsubsection{Autres questions discutées}

\section{3.1. Articulation synchronie / diachronie}

Cette thématique sera abordée dans un exposé indépendant de Franz Rainer. En complément des conclusions de ce dernier, on signalera le réinvestissement de la notion de paradigme flexionnel par la diachronie, dont certains travaux ont montré qu'il devait être vu comme une métamarque régularisatrice (Maiden).

\section{Général}

Maiden Martin. 1992. "Irregularity as a determinant of morphological change". Journal of Linguistics 28:285-312.

Maiden Martin. 2001. "What sort of thing is a derivational affix? Diachronic evidence from Rominian and Spanish suffixes". Yearbook of Morphology 1999:25-52. 
Rainer Franz. 2004. "L'origine dei nomi di strumento italiani in -tore". In Analecta homini universali dicata. Arbeiten zur Indogermanistik, Linguistik, Philologie, Politik, Musik und Dichtung. Festschrift für Oswald Panagl zum 65. Geburtstag, Krisch T., T. Linder \& U. Müller (eds). 399-424. Stuttgart: Verlag Hans-Dieter Heinz / Akademischer Verlag Stuttgart.

Rainer Franz. 2005. "Semantic change in word formation". Linguistics 43 2:415-441.

Rainer Franz. 2005. "Noms d'instruments / de lieu en -tor dans la Galloromania". Vox romanica $64: 121-140$.

\section{Domaine français}

Amiot Dany \& Dal Georgette. En préparation. "L'autonomie de la morphologie vue du côté de la grammaticalisation", Louvain, série «Mémoires de la Société Linguistique de Paris », Peeters Publishers.

Amiot Dany \& De Mulder Walter. 2002. "De l'adverbe au préfixe en passant par la préposition: un phénomène de grammaticalisation? ". Lingvisticae Investigationes XXV/2 : 247-273.

Amiot Dany \& De Mulder Walter. à paraître. "Un long fleuve pas si tranquille. De l'adverbe / préposition au préfixe : le cas de après(-) et arrière(-)", Langue française.

Roché Michel. 2005. "La dérivation en -ier(e) en ancien français". Lexique 17 : 55-96.

Rainer Franz. 2003. "L'intégration des composés latin du type aurifer en français". In Les unités morphologiques, Vol. 3, Silexicales, Fradin B., G. Dal, N. Hathout, F. Kerleroux, M. Plénat \& M. Roché (eds). 151-168. Villeneuve d'Ascq: SILEX: CNRS \& Université Lille 3.

Rainer Franz. 2007. "Frz. mammifère oder: habent sua fata et termini technici". Zeitschrift für französische Sprache 117 1:14-24.

\section{3.2. Morphologie non conventionnelle}

La possibilité d'altérer les lexèmes existants à des fins expressives existe dans beaucoup de langues, sinon dans toutes. Dans la mesure où nombre de ces formes se retrouvent dans le lexique, la question de leur formation et, du coup, de leur rapport avec les procédés permettant de former de manière régulière des unités ayant vocation à devenir des unités lexicales se pose. Parmi ces procédés non conventionnels, il faut compter les accourcissements (apocope e.g. prof < professeur, aphérèse blème < problème), la réduplication (fifille < fille), les langages secrets (verlan, loucherbem, javanais), les mots-valises (e.g. foultitude). Ces phénomènes sont particulièrement répandus en français et ont donné lieu à plusieurs études récentes.

\section{Général}

Dressler Wolfgang U. 2000. "Extragrammatical vs. marginal morphology". In Marginal and extragrammatical morphology, Doleschal U. \& A. Thornton (eds). 1-10. München: Lincom Europa. 
Dressler Wolfgang U. 1994. "Evidence from the first stages of morphology acquisition for linguistic theory: extragrammatic morphology and diminutives". Acta Linguistica Hafniensia 27 1:91-108.

\subsection{Domaine français}

Fradin Bernard. 2000. "Combining forms, blends and related phenomena". In Extragrammatical and marginal morphology, Doleschal U. \& A. Thornton (eds). 11-59. München: Lincom Europa.

Fradin Bernard, Fabio Montermini \& Marc Plénat. 2007 (à par.). "Morphologie grammaticale et extragrammaticale". In Aperçus de morphologie du français, Fradin B., F. Kerleroux \& M. Plénat (eds). 00-00. Saint-Denis: Presses Universitaires de Vincennes.

Kerleroux Françoise. 1997. "L'Apocope et les déverbaux". Cahiers de grammaire 22:155-186.

Kerleroux Françoise. 1999. "Sur quelles bases opère l'apocope?" In Morphologie des dérivés évaluatifs, Vol. 2, Silexicales, Corbin D., G. Dal, B. Fradin, B. Habert, F. Kerleroux, M. Plénat \& M. Roché (eds). 95-106. Villeneuve d'Ascq: Publication de l'UMR 8528 - CNRS \& Université de Lille 3.

Plénat Marc. 1996. "Les dérivés en -Vche. Première partie: analyse". Toulouse: PRC Communication Homme-Machine.Plénat, Marc. 1996. Les dérivés en -Vche. Première partie: analyse Technical PRC Communication Homme-Machine. Toulouse.

Plénat Marc. 1995. "Une approche prosodique de la morphologie du verlan". Lingua 95 1:97-129.

Plénat Marc \& Pancho Solares Huerta. 2006. "Domi, Seb, Flo et toute la famille (Lettre à Jean-Pierre Maurel sur les hypocoristiques français formés par apocope)". Cahiers de grammaire 30:339-357.

Roché Michel. 2008. "Quelques exemples de morphologie non conventionnelle dans les formations construites à partir d'un mot en -ouille(r)". In La raison morphologique. Hommage à la mémoire de Danielle Corbin, Vol. 27 Linguisticae Investigationes Supplementa, Fradin B. (ed). 215-238. Amsterdam / Philadelphie: John Benjamins.

\subsubsection{Nouveaux regards}

L'abandon de la notion de règle et de dérivation, comme séquence d'instructions s'appliquant à un input, a aussi eu des répercussions en morphologie. Cela s'est traduit directement, par la reformulation des règles sous forme de contraintes, ou de manière plus indirecte, par la prise en compte de la pression du lexique existant sur les possibilités de formation de lexèmes nouveaux. Le blocage est la manifestation la plus évidente et la plus anciennement repérée de cette pression. 


\section{4.1. Règles / contraintes}

\section{Général}

Barker Chris. 1998. "Episodic -ee in English: A thematic role constraint". Language 74 4:695-727.

Burzio Luigi. 2002. "Surface-to-surface Morphology: when your representation turn into Constraints". In Many Morphologies, Boucher Paul (ed). 142-177. Somerville, Mass.: Cascadilla Press.

Hay Jennifer \& Ingo Plag. 2004. "What constraints possible suffix combinations? On the interaction of grammatical and processing restrictions in derivational morphology?" Natural Languages \& Linguistic Theory 22 3:565-596.

Peperkamp Sharon. 1995. "Prosodic Constraints in the Derivational Morpholgy of Italian". Yearbook of Morphology 1994:207-244.

Plag Ingo. 1999. Morphological Productivity. Structural Constraints in English Derivation. Berlin / New York: Mouton de Gruyter.

Domaine français

Plénat Marc. 1993. "Observations sur le mot minimal français. L'oralisation des sigles". In De natura sonorum, Laks B. \& M. Plénat (eds). 143-172. Saint-Denis: Presses universitaires de Vincennes.

Plénat Marc. 1997. "Analyse morpho-phonologique d'un corpus d'adjectifs en -esque". French Language Studies 7:163-179.

Plénat Marc. 1998. "De quelques paramètres intervenant dans l'oralisation des sigles en français". Cahiers d'Etudes Romanes 9:27-52.

Plénat Marc. 1999. "Prolégomènes à une étude variationniste des hypocoristiques à redoublement en français". Cahiers de grammaire 24:183-219.

Plénat Marc. 2005. "Rosinette, cousinette, putinette, starlinette, chipinette: décalage, infixation et épenthèse devant -ette". In Questions de classification en linguistique: méthodes et descriptions. Mélanges offerts au Professeur Christian Molinier, Choi-Jonin I., M. Bras, A. Dagnac \& M. Rouquier (eds). 275-298. Bern / Berlin: Peter Lang.

Plénat Marc. 2007 (à par.). "Les contraintes de taille". In Aperçus de morphologie du français, Fradin Bernard, Françoise Kerleroux \& Marc Plénat (eds). 00-00. Saint-Denis: Presses Universitaires de Vincennes.

Plénat Marc \& Stéphanie Lignon. 2007 (à par.). "Echangisme suffixal et contraintes phonologiques". In Aperçus de morphologie du français, Fradin B., F. Kerleroux \& M. Plénat (eds). 00-00. Saint-Denis: Presses Universitaires de Vincennes.

Plénat Marc \& Michel Roché. 2003. "Prosodic Constraints on Suffixation in French". In Topics in Morphology. Selected papers from the Third Mediterranean Morphology Meeting (Barcelona, September 20-22, 2001), Booij Geert, Janet De Cesaris, Sergio Scalise \& Angela Ralli (eds). 285-299. Barcelona: IULAUniversitat Pompeu Fabra. 
Rainer Franz. 2005. "Constraints on productivity". In Handbook of word-formation, Štekauer Pavol \& Rochelle Lieber (eds). 335-352. Dordrecht: Springer.

\section{4.2. Blocage}

\section{Général}

Kiparsky Paul. 2005. "Blocking and periphrasis in inflectional paradigms". Yearbook of Morphology 2004:113-135.

Rainer Franz. 1989. "Towards a theory of blocking: the case of Italian and German quality nouns". Yearbook of Morphology 1988:155-185.

Scalise Sergio, Marco Ceresa, Marina Drigo, Maria Gottardo \& Irene Zanier. 1983. "Sulla nozione di 'Blocking' in morfologia derivazionale". Lingua e stile XVIII 2:243-269.

\section{Domaine français}

Dal Georgette \& Namer Fiammetta. 2003. "Complex words vs. phrases: the case of causative verbs in French". In G. Booij, J. De Cesaris, A. Ralli \& S. Scalise (eds), Topics in Morphology. Selected papers from the Third Mediterranean Morphology Meeting (Barcelona, september 20-22, 2001), Barcelona, Institut Universitari de lingüistica aplicada, Universitat Pompeu Fabra, pp. 129-148.

\section{4.3. Influence du lexique existant et dérivation paradigmatique}

La création de dérivés par analogie à partir de la mise en série de formes lexicales existantes est un autre phénomène qui a suscité l'intérêt ces dernières années. La dérivation paradigmatique, dans laquelle une forme existante et non plus un radical sert de base, peut être vue comme une instance grammaticalisée du même phénomène.

\section{Général}

Becker Thomas. 1993. "Back-formation, cross-formation, and 'bracketing paradoxes' in paradigmatic morphology". Yearbook of Morphology 1993:1-25.

Booij Geert. 2005. "Compounding and derivation. Evidence for Construction Morphology". In Morphology and its Demarcations, Dressler W. U., D. Kastovsky, O. E. Pfeiffer \& F. Rainer (eds). 109-132. Amsterdam / Philadelphia: John Benjamins.

Neuvel Sylvain. 2001. "Pattern analogy vs. word-internal syntactic structure in WestGreenlandic: Towards a functional definition of morphology". Yearbook of Morphology 2000:253-278.

van Marle Jaap. 1985. On the Paradigmatic Dimension of Morphological Creativity. Dordrecht: Foris Publications. 


\section{Domaine français}

Amiot Dany (en préparation), "Analogy vs. Rules: How can diachronic and synchronic perspectives be made to work together?". 6th Mediterranean Morphology Meeting, Ithaque. 27-30 septembre 2007.

Dal Georgette. 2003. "Analogie et lexique construit: quelles preuves?" Cahiers de grammaire 28:9-30. 